\title{
Knowledge Graph and Its Applications in MOOC and SPOC
}

\author{
Yunyan Liao ${ }^{1}$, Qing Huang ${ }^{1}$, Changjing Wang ${ }^{1}$, Zhengkang Zuo ${ }^{1}$, Yuan Wang ${ }^{2}$, Qiang $\mathrm{Yu}^{3}$ \\ ${ }^{1}$ Computer and Information Engineering College,Jiangxi Normal University,Nanchang, China \\ ${ }^{2}$ School of software,Jiangxi Normal University,Nanchang, China \\ ${ }^{3}$ Primary school affiliated to jiangxi normal universityNanchang, China
}

Keywords: Knowledge Graph, Mooc and Spoc, Education Big Data.

Abstract: As the core driving force to promote the development of artificial intelligence, knowledge graph provides a new power for education and teaching in the era of education information 2.0.Flipped classroom has achieved a significant change in the way of teaching and learning, but it lacks the process of constructing individual knowledge of learners, the content and relevance of learning generation. The attention of group wisdom on the implicit content such as the form and degree of individual support and its visualization. This paper introduces the knowledge of knowledge graph, and then Application of knowledge graphping in MOOC and SPOC.

\section{Introduction}

In the "Internet + " era, the traditional on-site learning mode in schools, which remains unidirectional and face-to-face can no longer meet the needs of students growing up on the digital earth. At present, the fantasy of the future school has sounded. Blended Learning combining the advantages of traditional classroom learning and online Learning will become the mainstream of teaching in the future. Blended learning based on the online learning platform, can greatly enrich the contents of the course. And this the make online courses a systematized and optional knowledge that merges resources such as words, animation, video, exploratory task, virtual experiment, through which students determine their own learning contents and process, and even seek for customization content.

Knowledge Graph, known as the knowledge domain visualization or maping, in the field of library and information, is a series of graphs showing the relationship between evolution and structure for science knowledge, using visualization technology to describe knowledge resources as well as their carriers, and mining, analyzing, constructing, plotting and displaying knowledge and their inter-relationships.

\section{Knowledge about Knowledge Graphs}

Knowledge Graph is a modern theory which combines theories and methods in applied mathematics, graphics, information visualization technology, information science and citation analysis, co-occurrence analysis in metrology, using a visual map to visualize the core structure, development history, frontiers and overall knowledge structure of a discipline to achieve 
multidisciplinary integration. It provides practical and valuable reference for discipline research.(baike 2019-9-15)

As one of the important knowledge representation methods in the era of artificial intelligence, knowledge graph breaks the data isolation in different scenarios and provides basic support for the applications like search, recommendation, question and answer, widely applied in different tasks. It can be widely applied to different tasks. Compared with deep learning, knowledge in the knowledge graph is highly explanatory and can be precipitated, which is similar to human thinking.

Compared with concept map, knowledge graph and cognitive map, knowledge graph can express more extensive knowledge content and semantic correlation, and it is more automated. But through literature analysis we found that the current knowledge Graph in education is still in the preliminary exploration stage. Following are some problems existing in terms of knowledge granularity, adaptability, constructional method, etc: (1) knowledge granularity, the current knowledge nodes in knowledge Graph is used to represent concepts, knowledge points, or real entities. With vague granularity, it has not yet reached the smallest independent unit of knowledge, the knowledge yuan;(2)domain adaptability, there is a lack of pertinent and pragmatic context in education and teaching, which has not yet reflected the different level of knowledge cognition among individual learners, and failed to simulate and reflect the state of individual learners' cognition.(3) constructional method, the construction process relies too much on subject experts, lack of automation. The cognitive deviation of different experts on the same knowledge point makes it difficult to guarantee the scientificity and consistency.

Existing research on personalized learning recommendation mainly recommend from the learners' interest in learning, learning style, learning preferences to the user recommend related resources, lack of users learning state, leading knowledge point of interest may already know (and therefore can not push or less recommended), and are not interested in mastering knowledge is not enough, but need further more recommendation). Therefore, in order to realize personalized recommendation of learning resources in online smart learning, it is more important to recommend learning resources according to user portraits and knowledge relationship diagrams, which may better meet the needs of learners.

Educational knowledge graph directly shows the relationship between knowledge in the form of graph. Its practical value is to help learners master the internal connection between knowledge. The scientific arrangement of knowledge acquisition in the process of teaching and learning;Accurate retrieval of digital teaching resources

Personalized learning is an eternal theme of educational reform and development, and an important proposition of educational innovation and development in the era of big data and artificial intelligence.Personalized learning intelligent recommendation mainly recommends relevant resources from learners' learning interest, learning style, cognitive level and learning preference.Personalized learning recommendation mainly recommends relevant resources to users from the aspects of learners' learning interest, learning style, cognitive level and learning preference.Zhu tianyu proposed using cognitive diagnosis model to recommend personalized test questions.He ling proposes to recommend current and follow-up materials in real time according to learners' interests and learning progress.Jingyongjun realizes the push service of basic education net resources through collaborative filtering technology combining content and cooperation.AviSegal USES collaborative filtering algorithm to propose a new personalized modeling method, EDUrank.Walker applied the collaborative filtering algorithm to the recommendation of college students' test questions and student materials. Wu proposed using fuzzy tree theory to recommend learning resources.Liiane and Dascalua et al realized personalized resource push according to learning goals and learning paths.Karampripeis proposed using ontology to generate personalized learning plans and make recommendations;Cheng proposed a mixed recommendation strategy 
based on content and knowledge.Niu wenjuan USES the time sequence interactive behavior data to recommend the result that can reflect the preference of the target user to the user.Yang chao integrated project response theory and particle swarm optimization algorithm to recommend resources.Yang hebiao et al proposed a recommendation method based on knowledge association rules.Li hongyan studies the recommendation method of personalized learning resources according to learning objectives and learning preferences.Jiang changmeng constructed a knowledge acquisition model for students and proposed TopN personalized exercise recommendation algorithm.Wang jing used ontology method to construct knowledge system and improved user-based collaborative filtering recommendation algorithm to recommend resources.Dwivedi personalized tweets based on group similarity.

Compared with concept map, knowledge graph and cognitive map, knowledge graph can express more extensive knowledge content and semantic correlation, and it is more automated. But through literature analysis we found that the current knowledge Graph in education is still in the preliminary exploration stage. Following are some problems existing in terms of knowledge granularity, adaptability, constructional method, etc: (1) knowledge granularity, the current knowledge nodes in knowledge Graph is used to represent concepts, knowledge points, or real entities. With vague granularity, it has not yet reached the smallest independent unit of knowledge, the knowledge yuan;(2)domain adaptability, there is a lack of pertinent and pragmatic context in education and teaching, which has not yet reflected the different level of knowledge cognition among individual learners, and failed to simulate and reflect the state of individual learners' cognition.(3) constructional method, the construction process relies too much on subject experts, lack of automation. The cognitive deviation of different experts on the same knowledge point makes it difficult to guarantee the scientificity and consistency.

Existing research on personalized learning recommendation mainly recommend from the learners' interest in learning, learning style, learning preferences to the user recommend related resources, lack of users learning state, leading knowledge point of interest may already know (and therefore can not push or less recommended), and are not interested in mastering knowledge is not enough, but need further more recommendation). Therefore, in order to realize personalized recommendation of learning resources in online smart learning, it is more important to recommend learning resources according to user portraits and knowledge relationship diagrams, which may better meet the needs of learners.

\section{Application of Knowledge Graphping in Mooc and Spoc}

The Massive Open online Course (MOOC) provides us with an online learning model that has never existed before. Small Private Online Courses (SPOC) was born in 2013. It is a more exquisite and niche online open course type than MOOC. Knowledge mapping has the following functions in Mooc and SPOC teaching .

\subsection{Knowledge Base Construction}

Knowledge base is the most important basic resource base in the education industry. It shows the hierarchical relationship and connection between mooc and spoc courses in the form of graphs, which can form a centralized, accurate, complete, concise and clear knowledge network diagram. Such a diagram structure is often more helpful for students to quickly clarify the connections and differences between various knowledge points, which is of great help to master the overall structure of the course.Various resources in the knowledge base, such as video, learning materials and test questions, can be linked to the knowledge node in the knowledge graph.Then the corresponding resource map is constructed according to the relationship of knowledge graph. 


\subsection{Adaptive Learning}

Adaptive learning builds learner portraits according to the behavior of mooc and spoc learners and their mastery of knowledge, and then plans different learning paths through knowledge graphs to provide learners with personalized courses and exercises with difficulty and rhythm, so as to improve learners' learning efficiency and learning effect.

\subsection{Virtual Learning Assistant}

Virtual learning assistants provide mooc and spoc learners with personalized question-answering partners, teaching assistants, and other services, as well as access to a large amount of user data feedback. Timely problem feedback can greatly promote the improvement of learners' performance.

\section{Function of Knowledge Graph in Teaching}

\section{1 knowledge retrieval and visualization functions}

Provide knowledge retrieval and visualization functions at all levels, and Various multi-mode resources related to knowledge points can be obtained based on the map.To help students learn better. Among them, the application is mainly built a good knowledge graph as the object, not only to the attributes of each knowledge point and the presentation of multimodal information, and more importantly the knowledge of each The correlation between recognition points is also shown. In short, to

The whole knowledge graph is presented as a directed graph. In the inspection

When asking for a specific knowledge point, send a letter related to that knowledge point Information, resources, and other related knowledge points are also shown.

\subsection{Application of Intelligent Online Teaching}

Intelligent online teaching applications can be based on specific teaching objectives

Marking and content, based on the general knowledge graph, are generated for this section. The subgraph of the class.Focus on the knowledge points involved in the sub-graph. Explain the content, according to the relationship between knowledge points (foreword course, after Organize the content of classroom teaching. The figure

The multimodal information in spectrum also provides rich information for multimedia teaching Resources. These multimedia resources are mounted to the corresponding knowledge point, learn Students can still focus on atlas in independent learning and after-class review Make full use of these resources.

\subsection{Personalized Learning and Teaching Application}

The emphasis of personalized learning and teaching application lies in "personalization". That is, "teach students according to their aptitude". It is based on knowledge graphs that will be collected. The examination questions to the kind of resources as the question bank, according to the teaching content and teachers

Develop the test requirements, automatically generate the specified knowledge points of the classroom exercises. The examination paper. The system will automatically give students a score as well as the answer. The solution of every wrong question. Then according to each student in class practice situation Give feedback and summarize your knowledge. One aspect, for the teacher, can master all students do the situation

Situation, we all grasp the knowledge point is not good to talk about. In addition, when students review after class, they can report according to the feedback. Understand the study circumstance of oneself each knowledge point, in order to have in the light of review and practice sex. 


\section{Conclusion}

In this paper, knowledge graph is applied to mooc and spoc teaching in universities. Subject knowledge points are taken as entities, attribute information and multimedia resources are extracted from textbooks, outline and network to construct subject knowledge graph.With knowledge graph as the cornerstone, teachers can conduct more effective wisdom classroom teaching.In addition, knowledge retrieval and visualization based on the map, intelligent classroom teaching, personalized learning and teaching application can assist teachers in targeted teaching.And according to each student's learning situation gives the different evaluation result, realizes the true individuality independent study.

\section{References}

[1] Liao Yunyan est, research on application of SPOC flipped classroom in computer basic courses, the $13^{\text {th }}$ international conference on computer science \& education, August 18-11

[2] Jordan K. Initial trends in enrolment and completion of massive open online courses[J]. International Review of Research in Open \& Distance Learning, 2014,(1):133-160.

[3] $\mathrm{Wu} \mathrm{W}, \mathrm{Li} \mathrm{H}$, Wang $\mathrm{H}$, et al. Probase: a probabilistic taxonomy for text understanding. international conference on management of data, 2012:481-492

[4] Knewton Adaptive Learning Building the World 's Most Powerful Education Recommendation Engine [DB/OL]. [2016 -11 -22].https://www.knewton.com/wp -content/uploads/knewton -adaptive -learning -whitepaper.pdf

[5] Makoto Miwa, Mohit Bansa. End-to-End Relation Extraction Using LSTMs on Sequences and Tree Structures[C]/Proceedings of ACL, 2016:1-13

[6] Haiping Zhu,Feng Tian,Ke Wu,et al.A Multi - constraint Learning Path Recommendation Algorithm Based on Knowledge Map[J].Knowledge-Based Systems,2018(143):102-114.

[7] Edsurge.Five Key Elements of Personalized Learning[EB/OL].https://www.edsurge.com/n/2015 -04 -01 -five-key-elements-of-personalized-learning,2016-01-23. 